\title{
Neurodegenerative changes in early- and late-onset cognitive impairment with and without brain amyloidosis
}

Eddie C. Stage $\mathrm{Jr}^{1}$, Diana Svaldi ${ }^{1,2}$, Meredith Phillips ${ }^{3}$, Victor Hugo Canela ${ }^{1}$, Tugce Duran ${ }^{4}$, Naira Goukasian ${ }^{5}$, Shannon L. Risacher ${ }^{6}$, Andrew J. Saykin ${ }^{6,7,8}$, Liana G. Apostolova ${ }^{1,6,7,8^{*}}$ (D) and for the Alzheimer's Disease Neuroimaging Initiative

\begin{abstract}
Background: A substantial number of patients clinically diagnosed with Alzheimer's disease do not harbor amyloid pathology. We analyzed the presence and extent of tau deposition and neurodegeneration in amyloid-positive (AD) and amyloid-negative (nonAD) ADNI subjects while also taking into account age of onset ( $<$ or $>65$ years) as we expected that the emerging patterns could vary by age and presence or absence of brain amyloidosis.

Methods: One hundred and ten early-onset AD (EOAD), 121 EOnonAD, 364 late-onset AD (LOAD), and 175 LOnonAD mild cognitive impairment (MCI) and dementia (DEM) subjects were compared to 291 ADNI amyloidnegative control subjects using voxel-wise regression in SPM12 with cluster-level family-wise error correction at $p_{\text {FWE }}<0.05$ ). A subset of these subjects also received ${ }^{18} \mathrm{~F}$-flortaucipir scans and allowed for analysis of global tau burden.

Results: As expected, relative to LOAD, EOAD subjects showed more extensive neurodegeneration and tau deposition in $A D$-relevant regions. EOnonAD $D_{M C I}$ showed no significant neurodegeneration, while EOnon $A D_{D E M}$ showed bilateral medial and lateral temporal, and temporoparietal hypometabolism. LOnonAD $\mathrm{MCI}_{\text {and }}$ LOnonAD $D_{D E M}$ showed diffuse brain atrophy and a fronto-temporo-parietal hypometabolic pattern. LOnonAD and EOnonAD subjects failed to show significant tau binding.
\end{abstract}

Conclusions: LOnonAD subjects show a fronto-temporal neurodegenerative pattern in the absence of tau binding, which may represent underlying hippocampal sclerosis with TDP-43, also known as limbic-predominant age-related TDP-43 encephalopathy (LATE). The hypometabolic pattern observed in EOnonAD DEM seems similar to the one observed in $E O A D_{M C 1}$. Further investigation into the underlying etiology of EOnonAD is warranted.

Keywords: Early onset, Late onset, Alzheimer's disease, AD, MRI, PET, Tau, Neurodegeneration, Hippocampal sclerosis, Limbic-predominant age-related TDP-43 encephalopathy, LATE

\footnotetext{
* Correspondence: lapostol@iu.edu

'Department of Neurology, Indiana University School of Medicine, 355 W 16th Street, Suite 4022, Indianapolis, IN 46202, USA

${ }^{6}$ Department of Radiology and Imaging Sciences, Center for Neuroimaging, Indiana University School of Medicine, Indianapolis, IN, USA

Full list of author information is available at the end of the article
}

C C The Author(s). 2020 Open Access This article is licensed under a Creative Commons Attribution 4.0 International License, which permits use, sharing, adaptation, distribution and reproduction in any medium or format, as long as you give appropriate credit to the original author(s) and the source, provide a link to the Creative Commons licence, and indicate if changes were made. The images or other third party material in this article are included in the article's Creative Commons licence, unless indicated otherwise in a credit line to the material. If material is not included in the article's Creative Commons licence and your intended use is not permitted by statutory regulation or exceeds the permitted use, you will need to obtain permission directly from the copyright holder. To view a copy of this licence, visit http://creativecommons.org/licenses/by/4.0/ The Creative Commons Public Domain Dedication waiver (http://creativecommons.org/publicdomain/zero/1.0/) applies to the data made available in this article, unless otherwise stated in a credit line to the data. 


\section{Introduction}

An estimated 5.8 million people in the USA are currently diagnosed with Alzheimer's disease (AD) [1]. Even when rendered by dementia experts, the clinical diagnosis of AD shows only modest accuracy [2]. Twenty-nine to $56 \%$ of clinically diagnosed $\mathrm{AD}$ patients are $\mathrm{AD}$ phenocopies that fail to show AD pathology upon postmortem examination [2]. With the development of amyloid tracers for positron emission tomography (PET), we can now readily distinguish true AD cases from amyloid-negative AD phenocopies (nonAD).

Ninety-seven percent of all AD cases have symptom onset at the age of 65 or older and are classified as "late-onset" (LO), while the remaining 3\% have symptom onset before the age of 65 and are termed "early-onset" (EO) [1, 3 , 4]. Pathologically, patients who are younger at disease onset show greater pathological burden [5-9]. Magnetic resonance imaging (MRI), ${ }^{18} \mathrm{~F}$-fluorodeoxyglucose (FDG) PET, and ${ }^{18}$ F-flortaucipir PET (tau PET) studies have shown that EO Alzheimer's subjects (EOAD) have more extensive atrophy, hypometabolism, and tau burden compared to LO Alzheimer's subjects (LOAD) [10-14]. More advanced pathologic burden in EOAD has been associated with more aggressive clinical course and is more likely to have an atypical presentation [15-18]. To our knowledge to date, the imaging biomarker profiles of early-onset nonAD (EOnonAD) have not been studied.

The Alzheimer's Disease Neuroimaging Initiative (ADNI) is a multisite, longitudinal study that collects standardized imaging, genetic, clinical, and fluid biomarkers from clinically diagnosed amnestic mild cognitive impairment (MCI), clinically diagnosed Alzheimer's dementia (DEM), and cognitively normal $(\mathrm{CN})$ control subjects as a part of a global research effort to better understand LOAD. While the majority of ADNI subjects are older than 65 years, ADNI contains a sizeable cohort of amnestic EO MCI or DEM subjects (age of symptom onset <65 years). The addition of amyloid imaging in the ADNI-GO/2 funding stages allowed researchers to ascertain the amyloid PET status of all ADNI participants and provided researchers the opportunity to study the biomarker-validated AD and nonAD phenocopies in greater detail.

In this study, our aim was to ascertain the extent and severity of tau and neurodegenerative pathology measured with tau PET, FDG PET, and MRI in EO and LO ADNI cohorts stratified by amyloid status as follows: EOAD MCI and DEM (EOAD MCI $_{\text {EOAD }}$ DEM), EOnonAD MCI and DEM (EOnonAD $\left.{ }_{M C I} ; E_{\text {EOnonAD }}{ }_{\mathrm{DEM}}\right)$, LOAD MCI and DEM (LOAD $\left.{ }_{M C I} ; \mathrm{LOAD}_{\mathrm{DEM}}\right)$, and LOnonAD MCI and DEM (LOnonAD $\left.{ }_{M C I} ; L_{\text {LOnon }} \mathrm{DD}_{\mathrm{DEM}}\right)$. We hypothesized that EOAD and EOnonAD subjects would have more severe neurodegeneration and greater tau burden relative to their LO counterparts, indicative of the greater disease burden likely required to have equivalent impairment to the significantly older LO group. We also hypothesized that non AD cases would have a nonAD-like pattern of neurodegeneration.

\section{Materials and methods Subjects}

Data used in the preparation of this article were obtained from the ADNI database (adni.loni.usc.edu). ADNI was launched in 2003 as a public-private partnership, led by Principal Investigator Michael W. Weiner, MD. The primary goal of ADNI has been to test whether serial MRI, PET, other biological markers, and clinical and neuropsychological assessments can be combined to accurately measure and predict the progression of MCI and Alzheimer's dementia. ADNI has undergone three complete funding cycles to date, ADNI1, ADNI-GO, and ADNI2, and is now in the ADNI3 cycle. ADNI-GO, ADNI2, and ADNI3 included ${ }^{18}$ F-florbetapir amyloid PET imaging.

The clinical and biomarker characteristics of the ADNI cohort have been previously published [19]. ADNI has enrolled clinically diagnosed $\mathrm{CN}$, amnestic $\mathrm{MCI}$, and amnestic DEM subjects. Probable AD DEM diagnosis is based on the National Institute of Neurological and Communicative Disorders and Stroke and the AD and Related Disorders Association (NINCDS-ADRDA) criteria [20]. Probable AD DEM subjects were 56 to 90 years old at enrollment, scored between 20 and 26 on the Mini-Mental State Examination (MMSE) [21] and 0.5-1 on the Clinical Dementia Rating (CDR) global score [22]. Subjects diagnosed as amnestic MCI ranged from 55 to 91 years old at enrollment, had no significant functional impairment, scored between 24 and 30 on the MMSE, had a global CDR of 0.5 (memory score $\geq$ 0.5), and had impairment on Wechsler Memory Scale - Logical Memory II test [23]. CN subjects had MMSE between 24 and 30 and a global CDR of 0 and did not meet criteria for MCI or DEM. Subjects were excluded due to inability to undergo MRI or if they had other neurological disorders, active depression, or history of psychiatric diagnosis, alcohol, or substance dependence within the past 2 years, less than 6 years of education or were not fluent in English or Spanish. The full list of inclusion/exclusion criteria may be accessed in the online ADNI protocol (http://www.adni-info.org/Scientists/ADNIStudyProcedures.html). Written informed consent was obtained from all participants, and the institutional review board (IRB) at all ADNI sites have reviewed and approved ADNI data collection protocol.

For our analysis, we used 231 EO subjects with reported age of symptom onset $\leq 65$ years from the ADNI database with available ${ }^{18} \mathrm{~F}$-florbetapir amyloid PET or CSF A $\beta$ data (219 of the 231 received ${ }^{18} \mathrm{~F}$-Florbetapir PET, while the remaining 12 had CSF A $\beta$ data). One hundred seventythree EO subjects met criteria for MCI and 58 for DEM. Sixty MCI and 50 DEM were amyloid-positive ${\left(E^{-O A D}\right.}_{\mathrm{MCI}}$ and $\left.\mathrm{EOAD}_{\mathrm{DEM}}\right)$, and 113 and 8, respectively, were 
amyloid-negative (EOnon $\mathrm{AD}_{\mathrm{MCI}}$ and $E$ Onon $\left.A D_{\mathrm{DEM}}\right)$ based on previously validated ${ }^{18} \mathrm{~F}$-florbetapir global means standard uptake volume ratio (SUVR) cut-off of 1.17 [24] or a CSF $A \beta_{1-42}$ level $<192 \mathrm{pg} / \mathrm{ml}$ [25]. The subset with tau PET ( ${ }^{18}$ F-Flortaucipir) included $10 \mathrm{EOAD}_{\mathrm{MCl}}, 7$ EOAD $_{\mathrm{DEM}}, 38$ EOnonAD $_{\mathrm{MCI}}$, and $3 \mathrm{EOnonAD}_{\mathrm{DEM}}$.

LO subjects included had either ${ }^{18} \mathrm{~F}$-florbetapir amyloid PET (488/539 subjects) or CSF A $\beta$ data (51/539 subjects), and all had FDG PET and MR imaging. Special care was taken to ensure that no LO diagnostic group was significantly more or less cognitively impaired than their EO counterpart (measured by MMSE) by removing outlier subjects, resulting in 539 subjects. Three hundred sixty-seven met criteria for MCI and 172 for DEM. Two hundred sixteen LO $\mathrm{MCI}$ and $148 \mathrm{LO}$ DEM were amyloid-positive $\left(\mathrm{LOAD}_{\mathrm{MCI}}\right.$ and $L O A D_{\text {DEM }}$ ), while $151 \mathrm{LO} \mathrm{MCI}$ and 24 LO DEM were amyloid-negative (LOnon $A D_{M C I}$; LOnonAD $D_{\mathrm{DEM}}$ ). The subset with tau PET included $53 \mathrm{LOAD}_{\mathrm{MCl}}$, $27 \mathrm{LOAD}_{\mathrm{DEM}}, 51$ LOnonAD $\mathrm{D}_{\mathrm{MCI}}$, and 2 LOnonAD $\mathrm{DEM}_{\mathrm{DEM}}$. Due to the low numbers of $\mathrm{EO}$ and $\mathrm{LO}$ nonAD $\mathrm{DEM}_{\mathrm{DEM}}$ subjects with ${ }^{18} \mathrm{~F}$-flortaucipir scans (3 and 2, respectively), they were grouped together with the $\mathrm{EO}$ and $\mathrm{LOnonAD}_{\mathrm{MCI}}$, resulting in 41 EOnonAD and 53 LOnonAD subjects in the tau comparisons.

Since EO and LO groups cannot be directly compared because neurodegenerative changes associated with aging could inadvertently confound the results, we conducted two sets of analyses. We first compared each EO and LO group to the same $\mathrm{CN}$ comparison group comprised of the 291 amyloid-negative $(S U V R<1.17) \quad C N$ subjects within the age range of 55 to 90 years old. This comparison allowed for a straightforward interpretation of the effect sizes as a measure of disease impact. Next, we repeated the analyses comparing the LO and EO groups to only the older $(N=146)$ and younger half $(N=145)$ of $\mathrm{CN}$, respectively. The latter results are presented in Additional figures 1, 2, 3, and 4 and Additional tables $1 \& 2$.

\section{MRI and PET acquisition and analyses}

ADNI MRI and PET acquisition and preprocessing protocols can be found at www.adni-info.org. The MRI data acquisition and preprocessing have been previously described elsewhere [20]. We downloaded preprocessed MRI data from LONI IDA (https://ida.loni.usc.edu). Scans were spatially warped to Montreal Neurological Institute (MNI) space and segmented into gray matter (GM), white matter, and CSF components using voxel-based morphometry (VBM) in Statistical Parametric Mapping version 12 (SPM12), as described previously [26]. GM maps were normalized and smoothed using $10-\mathrm{mm}$ full-width half maximum (FWHM) Gaussian kernel, which yielded gray matter density (GMD) data. Intracranial volume (ICV) was also calculated using FreeSurfer version 5.1.

PET scanners across sites were held to rigorous qualifications, calibration, and normalization standards as described in detail elsewhere [22]. We downloaded preprocessed amyloid, FDG PET, and tau PET data from LONI IDA (https://ida.loni.usc.edu). The scans were already averaged, aligned to standard space, re-sampled to a standard image and voxel size $(2 \mathrm{~mm} \times 2 \mathrm{~mm} \times 2 \mathrm{~mm})$, and smoothed to a uniform resolution as previously described [27]. We aligned the images to the corresponding MRI scan from the same visit and normalized them to MNI space using parameters obtained from the MRI segmentation using SPM12. PET scans were intensity normalized to mean pons uptake for FDG, whole cerebellum for amyloid and cerebellar crus for tau PET, resulting in whole brain SUVR images as previously described $[28,29]$. To assign subjects into groups by amyloid status, we used an amyloid PET cutoff of SUVR $\geq$ 1.17 or CSF $\mathrm{A} \beta_{1-42}$ level $<192 \mathrm{pg} / \mathrm{ml}[24,25]$.

\section{Statistical analyses}

\section{Clinical and demographic analyses}

The statistical distribution of clinical and demographic characteristics (age, education, global CDR, MMSE, and amyloid PET mean global SUVR) were analyzed in SPSS version 24.2 using one-way ANOVA. ANOVA $p$ values are listed in the tables and Bonferroni-corrected multiple comparisons $p$ values are listed in the results text. APOE4 genotype and sex frequency comparisons were done using a chi-square test with two-sided $p$ values. The alpha value for all comparisons was $p<0.05$. In addition, a mixed effects model was generated in SAS 9.4 to comparing change in cortical $\left[{ }^{18} \mathrm{~F}\right]$-florbetapir SUVR to the CN group as a reference.

\section{Parametric mapping}

We used voxel-wise linear regression models in SPM12 to study the extent and severity of neurodegeneration and tau burden in $\mathrm{EO}$ and $\mathrm{LO} \mathrm{AD}$ and nonAD groups relative to $\mathrm{CN}$ while controlling for age, sex, and education. Additionally, in the MRI analyses, we covaried for MRI field strength (1.5 T vs. $3 \mathrm{~T}$ ) and ICV. Family-wise error (FWE) cluster-level correction was applied to correct for multiple comparisons with a significance threshold of $p<0.01$. Because side-by-side interpretation of significance maps generated with unequal sample sizes can be misleading, we also derived $\beta$-coefficient maps to demonstrate the effect sizes in each comparison, which are visualized using the MRIcrogl version 2.1 software.

\section{Results \\ AD analyses \\ Demographic comparisons}

The demographic, neuropsychological, and amyloid burden comparisons of the amyloid positive diagnostic groups relative to $\mathrm{CN}$ are shown in Table 1 . Our $\mathrm{CN}$ were significantly older than the EOAD $\mathrm{MCI}_{\mathrm{C}}$ and $\mathrm{EOAD}$ $D E M$ and significantly younger than the $L O A D_{M C I}$ and LOAD $_{\text {DEM }}$ subjects $(p<0.001$, both). The $\mathrm{CN}$ subjects 
Table 1 EOAD and LOAD demographic comparisons to CN. The comparisons were done using ANOVA and chi-square tests with two-sided $p$ values. The Bonferroni-corrected pairwise differences relative to CN are discussed in the "Results" section. Significant $p$ values $(<0.05)$ are bolded

\begin{tabular}{|c|c|c|c|c|c|c|c|}
\hline Variable & $\begin{array}{l}C N \\
(\boldsymbol{N}=291)\end{array}$ & $\begin{array}{l}\mathrm{EOAD}_{\mathbf{M C l}} \\
(\boldsymbol{N}=60)\end{array}$ & $\begin{array}{l}\text { EOAD } \\
(\boldsymbol{N}=50)\end{array}$ & $\boldsymbol{p}$ value & $\begin{array}{l}\mathrm{LOAD}_{\mathbf{M C l}} \\
(\boldsymbol{N}=216)\end{array}$ & $\begin{array}{l}\angle O A D_{\text {DEM }} \\
(\boldsymbol{N}=148)\end{array}$ & $\boldsymbol{p}$ value \\
\hline Age, years, mean (SD) & $74.3(6.4)$ & $65.4(6.0)$ & $64.7(6.3)$ & $<0.001$ & $76.4(5.8)^{* *}$ & $78.3(5.9)^{* *}$ & $<0.001$ \\
\hline Sex, male \% & 52.2 & 46.7 & 44.0 & 0.461 & 60.2 & 58.8 & 0.174 \\
\hline Education, years, mean (SD) & $16.7(2.6)$ & $16.7(2.8)^{*}$ & $15.6(2.4)^{*}$ & 0.022 & $15.8(2.8)$ & $15.4(3.0)$ & $<0.001$ \\
\hline$\%$ APOE $\varepsilon 4,0 / 1 / 2$ alleles & $77 / 22 / 1$ & $18 / 52 / 30$ & $26 / 38 / 36$ & $<0.001$ & $34 / 52 / 14$ & $26 / 56 / 18$ & $<0.001$ \\
\hline Global CDR, mean (SD) & $0.02(0.09)$ & $0.50(0.00)^{* * *}$ & $0.87(0.33)^{* * *}$ & $<0.001$ & $0.50(0.16)^{* * *}$ & $0.84(0.36)^{* * *}$ & $<0.001$ \\
\hline MMSE, mean (SD) & $29.0(1.3)$ & $27.8(1.8)^{* * *}$ & $22.5(3.3)^{* * *}$ & $<0.001$ & $27.4(1.9)^{* * *}$ & $23.0(2.8)^{* * *}$ & $<0.001$ \\
\hline $\begin{array}{l}\text { Global cortical }\left[{ }^{18} \mathrm{~F}\right] \text {-florbetapir } \\
\text { SUVR, mean (SD) }\end{array}$ & $1.03(0.06)$ & $1.41(0.15)^{*}$ & $1.48(0.13)^{*}$ & $<0.001$ & $1.43(0.17)^{*}$ & $1.47(0.16)^{*}$ & $<0.001$ \\
\hline $\begin{array}{l}\text { Change in cortical }\left[{ }^{18} \mathrm{~F}\right] \text {-florbetapir } \\
\text { SUVR referenced to } \mathrm{CN} \text { ( } p \text { value) }\end{array}$ & ref & $0.0155(0.0352)$ & $0.0079(0.5071)$ & N/A & $0.0078(0.1449)$ & $0.0003(0.9692)$ & N/A \\
\hline Tau scans, $N$ & 126 & 10 & 7 & N/A & 53 & 27 & N/A \\
\hline
\end{tabular}

${ }^{*} \mathrm{MCl}$ and DEM significantly different at $p<0.05$

${ }^{* *} \mathrm{MCl}$ and DEM significantly different at $p<0.01$

*** $\mathrm{MCl}$ and DEM significantly different at $p<0.001$

Table 2 EO vs. LO demographic comparisons. The comparisons were done using ANOVA and chi-square tests with two-sided $p$ values. Significant $p$ values $(<0.05)$ are bolded

\begin{tabular}{|c|c|c|c|c|c|c|}
\hline Variable & $\begin{array}{l}\mathrm{EOAD}_{\mathrm{MCI}} \\
(N=60)\end{array}$ & $\begin{array}{l}L^{2 O A D_{M C I}} \\
(N=216)\end{array}$ & $p$ value & $\begin{array}{l}\text { EOnonAD } \\
(N=113)\end{array}$ & $\begin{array}{l}\text { LOnonAD }_{M C I} \\
(N=151)\end{array}$ & $p$ value \\
\hline Age, years, mean (SD) & $65.4(6.0)$ & $76.4(5.8)$ & $<0.001$ & $65.5(5.8)$ & $77.6(6.2)$ & $<0.001$ \\
\hline Sex, male \% & 46.7 & 60.2 & 0.065 & 50.4 & 59.6 & 0.138 \\
\hline Education, years, mean (SD) & $16.7(2.8)$ & $15.8(2.8)$ & 0.021 & $16.2(2.5)$ & $16.3(2.5)$ & 0.848 \\
\hline$\%$ APOE e4, 0/1/2 alleles & $18 / 52 / 30$ & $34 / 52 / 14$ & 0.004 & $68 / 30 / 2$ & $86 / 13 / 2$ & 0.005 \\
\hline Global CDR, mean (SD) & $0.50(0.00)$ & $0.50(0.16)$ & 0.823 & $0.46(0.17)$ & $0.48(0.11)$ & 0.167 \\
\hline MMSE, mean (SD) & $27.8(1.8)$ & $27.4(1.9)$ & 0.229 & $28.6(1.5)$ & $28.4(1.6)$ & 0.175 \\
\hline $\begin{array}{l}\text { Global cortical }\left[{ }^{18} \mathrm{~F}\right]-\text { florbetapir } \\
\text { SUVR, mean (SD) }\end{array}$ & $1.41(0.15)$ & $1.43(0.17)$ & 0.315 & $1.03(0.08)$ & $1.01(0.09)$ & 0.115 \\
\hline Tau scans, $N$ & 10 & 53 & N/A & 38 & 51 & N/A \\
\hline Diagnostic group $(N)$ & $\begin{array}{l}\text { EOAD } \\
(N=50)\end{array}$ & $\begin{array}{l}\text { LOAD } D_{\mathrm{DEM}} \\
(N=148)\end{array}$ & $p$ value & $\begin{array}{l}\text { EOnon } A D_{\text {DEM }} \\
(N=8)\end{array}$ & $\begin{array}{l}\text { LOnonAD } D_{\mathrm{DEM}} \\
(N=24)\end{array}$ & $p$ value \\
\hline Age, years, mean (SD) & $64.7(6.3)$ & $78.3(5.9)$ & $<0.001$ & $66.3(5.8)$ & $79.4(5.8)$ & $<0.001$ \\
\hline Sex, male \% & 44.0 & 58.8 & 0.069 & 50.0 & 83.3 & 0.059 \\
\hline Education, years, mean (SD) & $15.6(2.4)$ & $15.4(3.0)$ & 0.668 & $15.6(3.5)$ & $15.6(3.0)$ & 1.000 \\
\hline$\% A P O E$ e $4,0 / 1 / 2$ alleles & $26 / 38 / 36$ & $26 / 56 / 18$ & 0.022 & $71 / 14 / 14$ & $83 / 13 / 4$ & 0.642 \\
\hline Global CDR, mean (SD) & $0.87(0.33)$ & $0.84(0.36)$ & 0.579 & $0.69(0.26)$ & $0.83(0.24)$ & 0.155 \\
\hline MMSE, mean (SD) & $22.5(3.3)$ & $23.0(2.8)$ & 0.283 & $23.0(2.4)$ & $23.6(1.9)$ & 0.453 \\
\hline $\begin{array}{l}\text { Global cortical }\left[{ }^{18} \mathrm{~F}\right]-\text { florbetapir } \\
\text { SUVR, mean (SD) }\end{array}$ & $1.48(0.13)$ & $1.47(0.16)$ & 0.616 & $1.04(0.08)$ & $1.01(0.10)$ & 0.485 \\
\hline Tau scans, $\boldsymbol{N}$ & 7 & 27 & N/A & 3 & 2 & N/A \\
\hline
\end{tabular}


were significantly less impaired (global CDR and MMSE), had significantly fewer APOE4 carriers, and had a significantly lower $\left[{ }^{18} \mathrm{~F}\right]$-florbetapir SUVR compared to all cognitively impaired $\mathrm{AD}$ groups $(p<0.001$, all). In addition, EOAD $D_{\mathrm{DEM}}$ and both $\mathrm{LOAD}_{\mathrm{MCI}}$ and $\mathrm{LOAD}_{\mathrm{DEM}}$ had significantly fewer years of education than the $\mathrm{CN}$ group ( $p=0.006, p<0.001$, and $p<0.001$, resp). When the data were split into younger and older subgroups, the effects remained the same, except that $L_{0 A D}$ MCI subjects were significantly younger $(p<0.001)$ and $\mathrm{LOAD}_{\text {DEM }}$ no longer were significantly older or younger than the $\mathrm{CN}$ group (Additional Table 1).

As expected, the EOAD ${ }_{M C I}$ and $E O A D_{D E M}$ subjects were significantly younger compared to the $\mathrm{LOAD}_{\mathrm{MCI}}$ and LOAD DEM $_{\text {groups }}(p<0.001$, Table 2 , top). Compared to $E O A D_{M C I}, L O A D_{M C I}$ had significantly fewer years of education $(p=0.021)$. The $\mathrm{EOAD}_{\mathrm{DEM}}$ and LOAD $_{\text {DEM }}$ groups showed similar education. Both EOAD groups had a significantly higher percentage of $A P O E \& 4$ homozygotes compared to LOAD subjects (MCI $p=0.004$, DEM $p=0.022$ ). There were no significant differences in global ${ }^{18} \mathrm{~F}$-florbetapir SUVR, global CDR, or MMSE between EO and LOAD groups.

Regional amyloid comparisons between the AD subjects showed a significant difference $(p=0.044)$ in parietal cortices and significant $(p=0.048)$ difference in temporal amyloid SUVR between EOAD $\mathrm{MCI}_{\mathrm{M}}$ and EOADDEM (Additional Table 2).

\section{Imaging comparisons}

The FWE cluster-level corrected maps of the MRI, FDG PET, and tau PET comparisons of EOAD and LOAD spectrum individuals to $\mathrm{CN}$ are shown in Fig. 1. The same analyses limited to only subjects with tau PET scans are shown in Additional Figure 1 while Additional Figure 2 displays comparisons of EOAD and LOAD to younger and older $\mathrm{CN}$ subgroups, respectively. The pattern of neurodegeneration and tau deposition seen in Fig. 1 and Additional Figures 1 and 2 are very similar discounting the probability of exaggerated age or selection bias.

MRI (Fig. 1, top panel) The EOAD $\mathrm{MCI}_{\text {group showed }}$ two significant clusters of atrophy in the left and right medial and lateral temporal and left frontal cortices relative to the CN group (left cluster: cluster size $k=45,797$, cluster $p_{\mathrm{FWE}}<0.001$; right cluster: $k=20,760$, cluster $\left.p_{\text {FWE }}=0.003\right)$. Compared to the $\mathrm{CN}$ group, the $\mathrm{LOAD}_{\mathrm{MCI}}$ cohort showed significant atrophy of the bilateral medial and lateral temporal, temporoparietal, insular, occipital, and frontal regions (single cluster $k=$ 688,646 , cluster $\left.p_{\mathrm{FWE}}<0.001\right)$. The $\mathrm{EOAD}_{\mathrm{MCI}}$ group visually showed a larger effect size (i.e., more severe atrophy) than $\mathrm{LOAD}_{\mathrm{MCI}}$ in overlapping regions (see $\beta$ coefficient maps in Fig. 1, top panel).

Both $E O A D_{D E M}$ and $L O A D_{D E M}$ showed extensive atrophy throughout the brain compared to $\mathrm{CN}$ (single clusters, $k_{\mathrm{EO}}=1,541,575, k_{\mathrm{LO}}=1,503,763$, cluster $p_{\mathrm{FWE}}<$ 0.001 for both). The significance and $\beta$-coefficient maps show a stronger effect size (i.e., more severe atrophy) in the $E O A D_{D E M}$ than the $\mathrm{LOAD}_{\mathrm{DEM}}$ group (see $\beta$ coefficient maps in Fig. 1, top panel).

FDG PET (Fig. 1, middle panel) Compared to $\mathrm{CN}$, $E O A D_{M C I}$ showed a significant hypometabolic cluster in bilateral medial and lateral temporal and lateral, temporoparietal cortices $\left(k=32,246\right.$, cluster $\left.p_{\mathrm{FWE}}<0.001\right)$. Additionally, there was a small cluster of hypometabolism in bilateral dorsolateral prefrontal cortex $(k=3396$, cluster $\left.p_{\mathrm{FWE}}<0.001\right)$. LOAD $\mathrm{MCI}_{\mathrm{M}}$ subjects showed hypometabolism of the bilateral inferior temporal, medial and lateral temporal, and parietal cortices as well as bilateral frontal cortex (single cluster, $k=94,307$, cluster $p_{\mathrm{FWE}}<0.001$, Fig. 1, middle panel).

Both the EOAD $D_{\text {DEM }}$ and $L O A D_{\text {DEM }}$ groups showed extensive hypometabolism relative to $\mathrm{CN}$ in bilateral parietal, temporal, and frontal lobes, as well as insular and cingulate cortices (single clusters, $k_{\mathrm{EO}}=148,701, k_{\mathrm{LO}}=$ 185,998 , cluster $p_{\mathrm{FWE}}<0.001$ for both). As with the MRI analysis, the $E O A D_{D E M}$ group showed a stronger effect size (i.e., more severe hypometabolism) than the LOADDEM group (see $\beta$-coefficient maps in Fig. 1, middle panel).

Tau PET (Fig. 1, bottom panel) These analyses were limited to the subset of individuals with available tau PET imaging. Compared to the $\mathrm{CN}$ group, the $\mathrm{EOAD}_{\mathrm{MCI}}$ group had a significant cluster of tau binding covering temporal, parietal, parietooccipital, and right frontal cortices $\left(k=74,981\right.$, cluster $\left.p_{\mathrm{FWE}}<0.001\right)$. An additional significant cluster of tau binding was present in the left prefrontal cortex $\left(k=9800\right.$, cluster $\left.p_{\mathrm{FWE}}<0.001\right)$. The LOAD $_{\text {MCI }}$ cohort showed tau deposition in a similar pattern (single cluster, $k=96,885$, cluster $p_{\text {FWE }}<0.001$ ). The beta coefficient maps demonstrated greater tau burden in $\mathrm{EOAD}_{\mathrm{MCI}}$ compared to $\mathrm{LOAD}_{\mathrm{MCI}}$ (see $\beta$-coefficient maps in Fig. 1, bottom panel).

$E O A D_{D E M}$ showed tau binding in all cortical regions save for the primary sensorimotor and visual cortices (single cluster, $k=157,966$, cluster $p_{\mathrm{FWE}}<0.001$ ). LOADDEM showed two significant clusters of tau binding-one in the posterior association cortices $(k=67,260$, cluster $\left.p_{\text {FWE }}<0.001\right)$ and a smaller one in the bilateral prefrontal cortices $\left(k=4931\right.$, cluster $\left.p_{\mathrm{FWE}}<0.001\right)$. The $\beta$ coefficient maps show much more severe and extensive tau deposition in $E O A D_{D E M}$ compared to LOAD $_{\text {DEM }}$ (see $\beta$-coefficient maps in Fig. 1, bottom panel). 


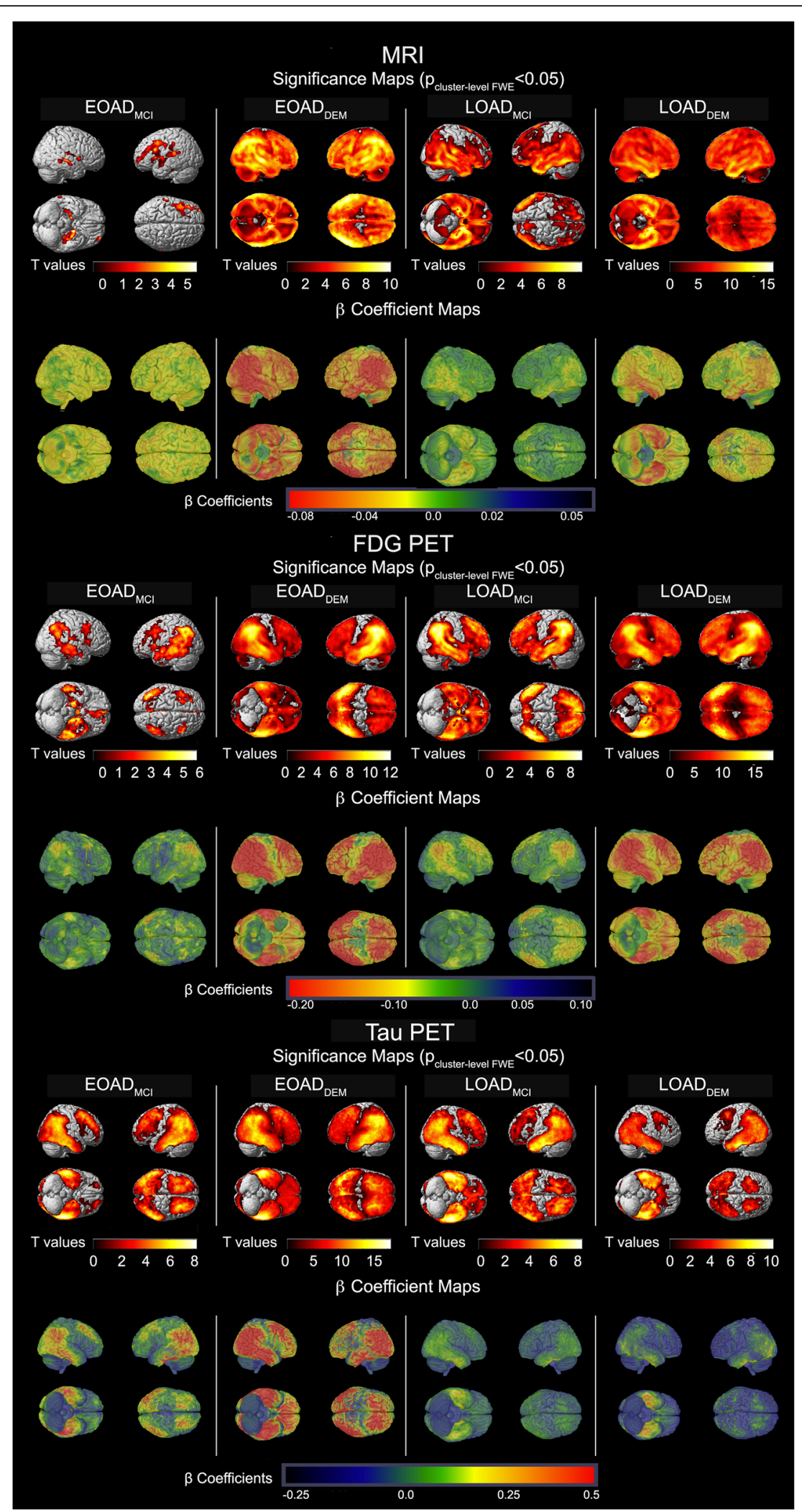

Fig. 1 MRI (top), FDG PET (middle), and tau PET (bottom) comparisons between the AD groups and CN. The significance maps show $p<0.05$ thresholded FWE cluster-level corrected results of EOAD MCI $(N=60)$, EOAD DEM $(N=50), \operatorname{LOAD}_{M C I}(N=216)$, and $\operatorname{LOAD}_{\mathrm{DEM}}(N=148)$ vs. $C N(N=291)$. The results displayed here are for all subjects with available scans in each modality 


\section{NonAD analyses}

\section{Demographic comparisons}

Direct comparisons of $\mathrm{CN}$ to EOnonAD $\mathrm{ECI}_{\mathrm{MCI}}$, LOno$\mathrm{nAD}_{\mathrm{MCI}}$, EOnonAD $\mathrm{DEM}_{\mathrm{DE}}$, and LOnonAD $\mathrm{DEM}_{\mathrm{DEM}}$ showed the expected significant difference in age, global CDR, and MMSE $(p<0.001$, Table 3$)$. Compared to CN, LOno$\mathrm{nAD}_{\mathrm{DEM}}$ had a greater proportion of men $(p=0.003)$ and lower education $(p=0.044)$. Even when split in younger and older subgroups, the age differences between $\mathrm{CN}$ and the respective disease groups remained significant with the exception of LOnon $\mathrm{AD}_{\mathrm{DEM}}$ (Additional Table 3).

By definition, EOnon $\mathrm{AD}_{\mathrm{MCI}}$ and $\mathrm{EOnon}_{\mathrm{AD}} \mathrm{D}_{\mathrm{DEM}}$ were significantly younger than the corresponding LOnonAD groups $(p<0.001)$. The EOnonAD $\mathrm{ACI}_{\mathrm{MCI}}$ group had a higher proportion of $A P O E \& 4$ carriers compared to the LOnonAD $\mathrm{ACI}_{\mathrm{MCI}}$ group $(p=0.005)$. There were no significant differences in sex, education, global CDR, MMSE, or global ${ }^{18} \mathrm{~F}$-florbetapir SUVR between the groups.

A closer look into regional amyloid differences among amyloid-negative subjects revealed significant differences between the LOnon $\mathrm{AD}_{\mathrm{DEM}}$ group and $\mathrm{CN}$, EOnonAD $\mathrm{ADC}_{\mathrm{MC}}$, EOnonAD $\mathrm{DEM}_{\mathrm{DE}}$, and LOnonAD $\mathrm{ACI}_{\mathrm{MCI}}$ in parietal SUVR $(p<$ 0.05 for all). No other region was significantly different.

\section{Imaging comparisons}

The FWE cluster-level corrected MRI, FDG PET, and tau PET comparison maps of the nonAD groups to $\mathrm{CN}$ are shown in Fig. 2. The same analyses limited to only subjects with tau PET scans are shown in Additional Figure 3, while Additional Figure 4 displays comparisons of EOnonAD and LOnonAD to younger and older $\mathrm{CN}$ subgroups, resp. The pattern of neurodegeneration and tau deposition seen in Additional Figure 4 is largely identical to the one in Fig. 2, with the exception of emerging tau deposition in bilateral frontal and right parietal lobes in EOnonAD when compared to the young $\mathrm{CN}$.

MRI (Fig. 2, top panel) EOnonAD showed no significant atrophy compared to the $\mathrm{CN}$ group. LOnon $\mathrm{AD}_{\mathrm{MCI}}$ showed extensive atrophy in the bilateral medial and lateral temporal, temporoparietal, parietooccipital, and frontal cortices (single cluster, $k=569,219$, cluster $\left.p_{\text {FWE }}<0.001\right)$. LOnonAD ${ }_{\text {DEM }}$ had similarly widespread atrophy showing two significant clusters-one in bilateral temporoparietal and frontal cortices $(k=602,716$, cluster $\left.p_{\text {FWE }}<0.001\right)$ and another in the cerebellum $(k=$ 13,494 , cluster $\left.p_{\text {FWE }}=0.020\right)$. The largest effect size was observed in LOnon $\mathrm{AD}_{\mathrm{DEM}}$ with greatest predilection for the medial and inferior temporal lobes (see $\beta$-coefficient maps in Fig. 2, top panel).

FDG PET (Fig. 2, middle panel) Compared to $\mathrm{CN}$, EOnon $\mathrm{AD}_{\mathrm{MCI}}$ showed no significant hypometabolism, while the EOnonAD ${ }_{\text {DEM }}$ group showed three significant clusters in left and right temporoparietal (left: $k=15,114$, cluster $p_{\mathrm{FWE}}<0.001$; right: $k=6104$, cluster $p_{\mathrm{FWE}}<$ 0.001 ) and bilateral frontal cortices (single cluster, $k=$ 3002 , cluster $\left.p_{\mathrm{FWE}}<0.001\right)$. LOnonAD $\mathrm{D}_{\mathrm{MCI}}$ showed a significant cluster of hypometabolism in bilateral temporal and prefrontal cortices $\left(k=24,278\right.$, cluster $\left.p_{\mathrm{FWE}}<0.001\right)$. A similar fronto-temporal pattern of hypometabolism was also observed in LOnon $\mathrm{AD}_{\mathrm{DEM}}$; however, it also extended to the parietal lobes (single cluster, $k=78,550$, cluster $\left.p_{\text {FWE }}<0.001\right)$.

Table 3 EOnonAD and LOnonAD demographic comparisons to CN. The comparisons were done using ANOVA and chi-square tests with two-sided $p$ values. The Bonferroni-corrected pairwise differences relative to CN are discussed in the "Results" section. Significant $p$ values $(<0.05)$ are bolded

\begin{tabular}{|c|c|c|c|c|c|c|c|}
\hline Variable & $\begin{array}{l}C N \\
(\boldsymbol{N}=291)\end{array}$ & $\begin{array}{l}\text { EOnonAD } \\
(\boldsymbol{N}=113)\end{array}$ & $\begin{array}{l}\text { EOnonAD }{ }_{\text {DEM }} \\
(\boldsymbol{N}=8)\end{array}$ & $\boldsymbol{p}$ value & $\begin{array}{l}\text { LOnonAD } \\
(\boldsymbol{N}=151)\end{array}$ & $\begin{array}{l}\text { LOnonAD }_{\text {DEM }} \\
(\boldsymbol{N}=24)\end{array}$ & $\boldsymbol{p}$ value \\
\hline Age, years, mean (SD) & $74.3(6.4)$ & $65.5(5.8)$ & $66.3(5.8)$ & $<0.001$ & $77.6(6.2)$ & $79.4(5.8)$ & $<0.001$ \\
\hline Sex, male \% & 52.2 & 50.4 & 50.0 & 0.944 & $59.6^{*}$ & $83.3^{*}$ & 0.008 \\
\hline Education, years, mean (SD) & $16.7(2.6)$ & $16.2(2.5)$ & $15.6(3.5)$ & 0.095 & $16.3(2.5)$ & $15.6(3.0)$ & 0.037 \\
\hline$\%$ APOE $\varepsilon 4,0 / 1 / 2$ alleles & $77 / 22 / 1$ & $68 / 30 / 2$ & $71 / 14 / 14$ & 0.028 & $86 / 13 / 2$ & $83 / 13 / 4$ & 0.173 \\
\hline Global CDR, mean (SD) & $0.02(0.09)$ & $0.46(0.17)^{* * *}$ & $0.69(0.26)^{* * *}$ & $<0.001$ & $0.48(0.11)^{* * *}$ & $0.83(0.24)^{* * *}$ & $<0.001$ \\
\hline MMSE, mean (SD) & $29.0(1.3)$ & $28.6(1.5)^{* * *}$ & $23.0(2.4)^{* * *}$ & $<0.001$ & $28.4(1.6)^{* * *}$ & $23.6(1.9)^{* * *}$ & $<0.001$ \\
\hline $\begin{array}{l}\left.\text { Global cortical [ }{ }^{18} \mathrm{~F}\right] \text {-florbetapir } \\
\text { SUVR, mean (SD) }\end{array}$ & $1.03(0.06)$ & $1.03(0.08)$ & $1.04(0.08)$ & 0.214 & $1.01(0.09)$ & $1.01(0.10)$ & 0.160 \\
\hline $\begin{array}{l}\text { Change in cortical }\left[{ }^{18} \mathrm{~F}\right] \text {-florbetapir } \\
\text { SUVR referenced to } \mathrm{CN} \text { ( } p \text { value) }\end{array}$ & ref & $\begin{array}{l}-0.0049 \\
(0.1835)^{*}\end{array}$ & $\begin{array}{l}-0.0387 \\
(\mathbf{0 . 0 1 7 5})^{*}\end{array}$ & N/A & $\begin{array}{l}-0.0099 \\
(\mathbf{0 . 0 0 9 1})\end{array}$ & $\begin{array}{l}0.0093 \\
(0.5425)\end{array}$ & N/A \\
\hline Tau scans, $\boldsymbol{N}$ & 126 & 38 & 3 & N/A & 51 & 2 & $\mathrm{~N} / \mathrm{A}$ \\
\hline
\end{tabular}

* $\mathrm{MCl}$ and DEM significantly different at $p<0.05$

${ }^{* * *} \mathrm{MCl}$ and DEM significantly different at $p<0.001$ 


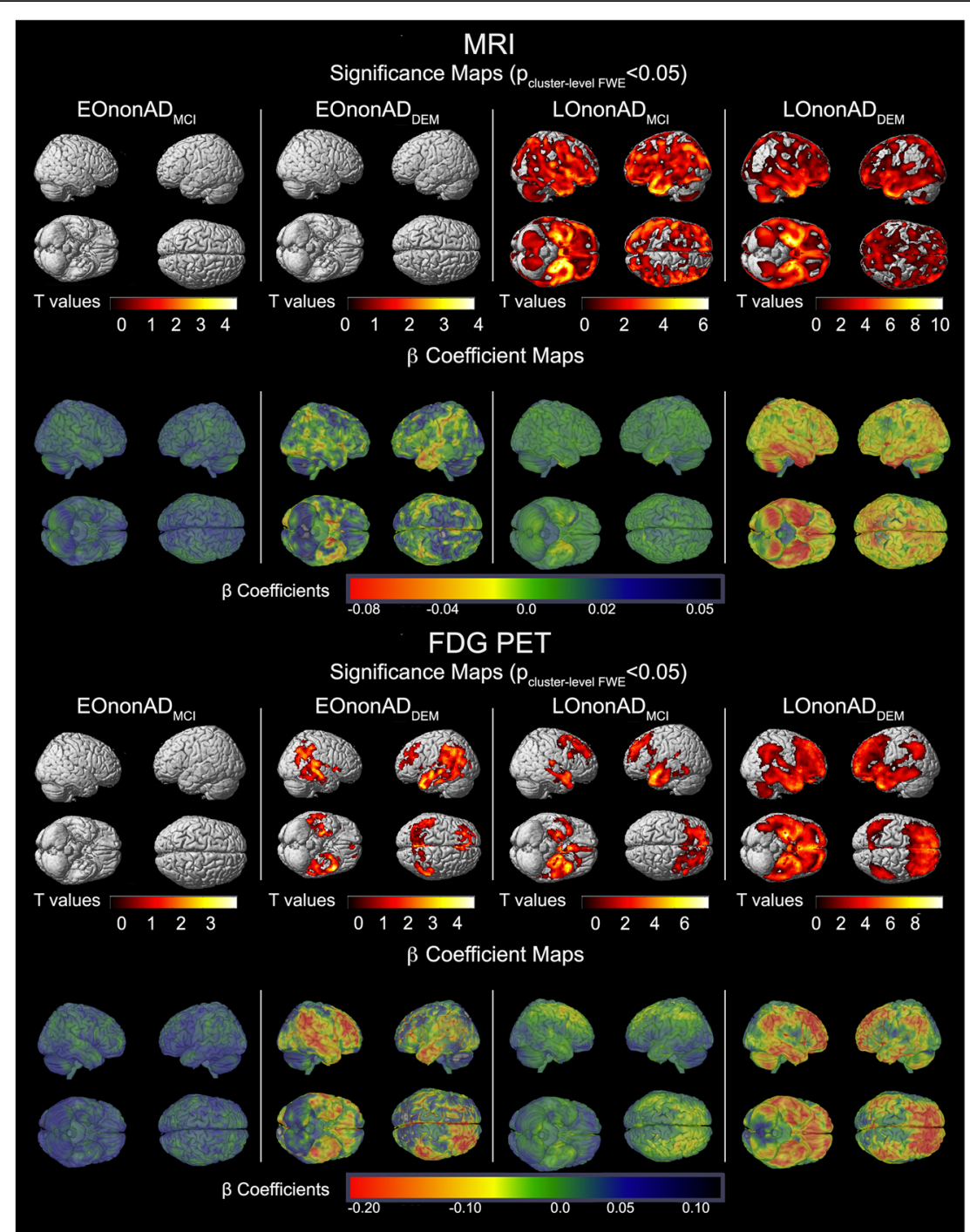

Tau PET

Significance Maps $\left(p_{\text {clusterlevel FWE }}<0.05\right)$
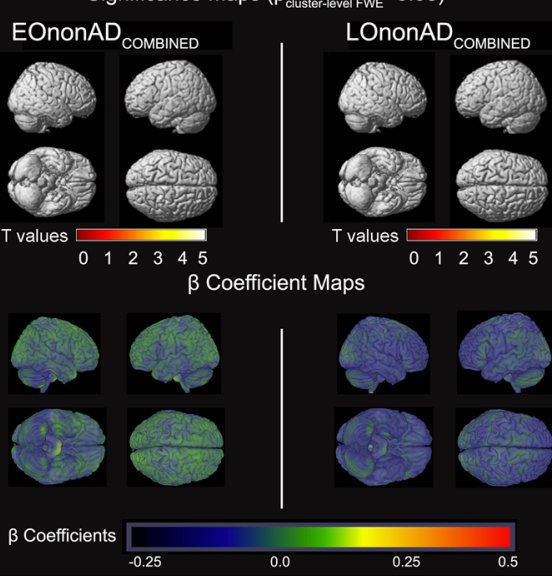

Fig. $2 \mathrm{MRI}$ (top), FDG PET (middle), and tau PET (bottom) comparisons between the nonAD groups and CN. The significance maps show $p<0.05$ thresholded FWE cluster-level corrected results of EOnonAD $D_{M C I}(N=113)$, EOnonAD $D E M(N=8)$, LOnonAD $D_{M C I}(N=151)$, and LOnonAD vs. CN $(N=291)$. The results displayed here are for all subjects with available scans in each modality 
Tau PET (Fig. 2, bottom panel) Due to the small sample sizes, the EOnonAD and LOnonAD groups were not split by disease stage. There was no significant tau binding in either EOnonAD or LOnonAD. As mentioned above, EOnonAD showed tau deposition in bilateral frontal (single cluster, $k=7215$, cluster $p_{\mathrm{FWE}}<0.001$ ) and right parietal lobes (single cluster, $k=4664$, cluster $p_{\mathrm{FWE}}<0.001$ ) when compared to young $\mathrm{CN}$ only (Additional Figure 4).

\section{Discussion}

The current study aimed to map neurofibrillary, structural, and metabolic differences between EO and LO MCI and DEM subjects stratified by amyloid positivity. As expected, we found that $E O A D_{M C I}$ and $E O A D_{D E M}$ subjects show more severe neurodegeneration and greater tau deposition compared to $\mathrm{LOAD}_{\mathrm{MCI}}$ and $\mathrm{LOAD}_{\mathrm{DEM}}$, respectively, a finding that is consistent with previous imaging reports $[5-11,14,18]$, and with the fact that $\mathrm{EO}$ individuals have a much more aggressive disease course $[15,18]$.

The availability of amyloid PET imaging or CSF A $\beta$ measurements allowed us to identify nonAD cases that were enrolled as Alzheimer's phenocopies. While we failed to find significant neurodegeneration in EOno$n A D_{M C I}$, we observed significant hypometabolism in EOnon $A D_{\mathrm{DEM}}$ in the absence of significant atrophy, a finding that could be indicative of synaptic dysfunction before cellular loss. The lack of findings, particularly in the EOnon $\mathrm{AD}_{\mathrm{MCI}}$ subjects, where the neurodegenerative changes are likely subtle, may be due to our inability to properly account for age-related degeneration (despite covarying for age during the analysis) when comparing directly to the $\mathrm{CN}$ subjects who are significantly older. In our EOnon $\mathrm{AD}_{\mathrm{DEM}}$ subjects, the lack of significant atrophy may be due to a relatively small sample size, as the beta-coefficient maps indicate a pattern of neurodegeneration similar to that seen in the FDG PET analysis. Furthermore, the EOnon $A D_{D E M}$ subjects may be a heterogeneous group of multiple etiologies, making detection of significant clusters of atrophy difficult.

LOnonAD cases showed pronounced atrophy and hypometabolism with greatest predilection for the temporal and frontal lobes. This pattern of neurodegeneration has been reported in primary age-related tauopathy (PART) and hippocampal sclerosis with TAR-DNA binding protein 43 (TDP-43) inclusions (HS-TDP-43) $[30,31]$. Both conditions are highly prevalent among the elderly with and without cognitive deficit [30, 31]; however, of these two, HS-TDP-43 (also known as limbic-predominant age-related TDP-43 encephalopathy (LATE) [32]) is the more likely etiology due to the lack of tau binding in the medial temporal lobes which is expected in PART. It is worth noting that though ${ }^{18} \mathrm{~F}$ - flortaucipir binds well to mature tangles in $3 R+4 R$ tauopathies, such as in AD and PART [33, 34], further post mortem studies are needed to say with confidence which tauopathy tau variants can reliably be bound with flortaucipir.

The hypometabolic pattern we observed in LOnonAD fits well with previous pathologic and imaging reports of LATE. TDP-43 inclusions and neurite deposits first appear in the hippocampal dentate granule cells, subiculum, and the amygdala [31, 35, 36]. In more advanced stages, TDP-43 pathology is also found in frontal and temporal neocortex $[31,35,36]$. TDP-43 pathology is extremely prevalent among cognitively impaired elderly and is the stand-alone pathology in $4.2 \%$ of these cases [31, 35, 37, 38]. Eighty-six percent of TDP-43-positive cases have HS-TDP-43 [31, 39-41]. HS-TDP-43 oftentimes show episodic and semantic memory dysfunction [42], explaining how they could easily be diagnosed clinically with AD. Individuals with HS-TDP-43 have greater hippocampal atrophy and greater cognitive impairment than those with HS without TDP-43 [35, 36, 39]. Additional support for our hypothesis that our LOnonAD subjects likely harbor HS-TDP-43 are the recent reports that HS-TDP-43 cases show hypometabolic changes in the medial and lateral temporal, posterior and middle cingulate, precuneus, and prefrontal cortex [43], similar to the FDG PET pattern we observed in LOnonAD $\mathrm{DCI}_{\mathrm{MCI}}$ and $\mathrm{LOnonAD}_{\mathrm{AD}}$. Similar hypometabolic and atrophy patterns involving medial and lateral temporal and prefrontal cortices were recently reported in two additional clinic-pathologic studies [44, 45].

An additional possibility is that some LOnonAD subjects may suffer from behavioral variant frontotemporal dementia (bvFTD). However, this is less likely given the mean age of our LOnonAD cohorts (77.6 and 79.4 years, resp) and their amnestic predominant presentation at enrollment as required by ADNI (see http://www.adni-info. org/Scientists/ADNIStudyProcedures.html). It is worth noting, however, that in rare cases ( $10 \%$ of pathologically confirmed bvFTD cases), patients presented with primarily amnestic symptoms and some studies have even reported as much as $25 \%$ of pathologically confirmed FTLD cases to have a disease onset after the age of 65 [46, 47].

Several strengths and limitations of our study should be noted. One of the strengths is the relatively large sample size of EO subjects available through ADNI. Additionally, ADNI employs meticulously standardized clinical and imaging data collection, which is routinely subjected to quality control. One of the limitations of our analyses is the cross-sectional design and the measurement of atrophy, which has a temporal component. This means that we are actually measuring differences in gray matter density, which implies atrophy, but is not synonymous. Longitudinal analyses are needed to assess 
atrophy and metabolic changes over time. Additionally, while the rigorous exclusion criteria employed in ADNI are typical of clinical trials, this renders the ADNI's population as not representative of the general population. Furthermore, there is very little post mortem data currently available for ADNI, which means diagnosis of AD largely lacks pathological verification. Finally, while we are including the EOnon $\mathrm{AD}_{\mathrm{DEM}}$ in our report for completeness, one must keep in mind that the number of subjects in this group is very small; thus, the findings should be interpreted with caution. Larger research studies such as the recently funded Longitudinal Earlyonset Alzheimer's disease Study (LEADS) which will use amyloid imaging and detect EOnonAD cases will be able to define the neurodegenerative pattern in this group.

\section{Conclusion}

In conclusion, our study found a similar neurodegenerative pattern between amnestic amyloid-positive EO and LO MCI and DEM subjects. These processes were more severe in the EO group indicating a more aggressive disease course. We also found that LOnonAD ${ }_{\mathrm{DEM}}$ subjects show anterior temporal neurodegeneration which might reflect the presence HS-TDP-43 or LATE. In the absence of reliable in vivo TDP-43 biomarker, the only feasible method of confirmation is through post-mortem examination of the brains. Other large research consortia such as the recently funded LEADS project will allow us the opportunity to systematically study EOAD and EOnonAD and further characterize these highly understudied disease states.

\section{Supplementary information}

Supplementary information accompanies this paper at https://doi.org/10. 1186/s13195-020-00647-w.

Additional Table 1. EOAD and LOAD demographic comparisons to the young and old CN groups, resp. The comparisons were done using ANOVA and chi-square tests with two-sided $p$-values. The Bonferronicorrected pairwise differences relative to $\mathbf{C N}$ are discussed in the Results section. Significant $p$-values $(<0.05)$ are bolded.

Additional Table 2. Regional amyloid PET (18F-Florbetapir) comparisons between amyloid positive subjects for frontal, cingulate, parietal and temporal cortices.

Additional Table 3. EOnonAD and LOnonAD demographic comparisons to the young and old CN groups, resp. The comparisons were done using ANOVA and chi-square tests with two-sided $p$-values. The Bonferroni-corrected pairwise differences relative to $\mathbf{C N}$ are discussed in the Results section. Significant $p$-values $(<0.05)$ are bolded.

Additional Table 4. Regional amyloid PET (18F-Florbetapir) comparisons between amyloid negative subjects for frontal, cingulate, parietal and temporal cortices.

Additional Figure 1. MRI (top), FDG PET (middle), tau PET (bottom) comparisons between the $A D$ and $C N$ groups restricted to only subjects with available tau PET scans. The significance maps show $p<0.05$ thresholded FWE cluster-level corrected results. of EOAD MCI $(N=10)$, $\operatorname{EOAD}_{\mathrm{DEM}}(N=7), \operatorname{LOAD}_{\mathrm{MCI}}(N=53)$ and LOAD DEM $(N=27)$ vs. $C N(N=$ 126).
Additional Figure 2. MRI (top), FDG PET (middle), tau PET (bottom) comparisons between young $C N$ and EOAD and old CN and LOAD groups. The significance maps show $p<0.05$ thresholded FWE clusterlevel corrected results of $\operatorname{EOAD}_{M C I}(N=60)$ and $\operatorname{EOAD}_{\mathrm{DEM}}(N=50)$ vs young $C N(N=145), \operatorname{LOAD}_{M C I}(N=216)$ and $\operatorname{LOAD}_{\mathrm{DEM}}(N=148)$ vs. old CN $(N=146)$.

Additional Figure 3. MRI (top), FDG PET (middle), tau PET (bottom) comparisons between the nonAD and CN groups restricted to only subjects with available tau PET scans. The significance maps show $p<$ 0.05 thresholded FWE cluster-level corrected results of EOnonAD $\mathrm{MCI}_{(\mathrm{N}}(N=$ 38), EOnonAD $\operatorname{DEM}_{(N=3), L\left(O n o n A D_{M C I}\right.}(N=51)$ and LOnonAD $D_{D E M}(N=2)$ vs. $C N(N=126)$.

Additional Figure 4. MRI (top), FDG PET (middle), tau PET (bottom) comparisons between young $\mathrm{CN}$ and EOnonAD and old $\mathrm{CN}$ and LOnonAD groups. The significance maps show $p<0.05$ thresholded FWE cluster-level corrected results of EOnonAD $D_{M C I}(N=113)$ and EOnonAD $(N=8)$ vs young $C N(N=145)$, LOnonAD $M C I=151)$ and LOnonAD $(N E M$ $(N=24)$ vs. old $C N(N=146)$.

\section{Abbreviations}

AD: Alzheimer's disease; ADNI: Alzheimer's Disease Neuroimaging Initiative; CDR: Clinical dementia rating; CN: Cognitively normal; DEM: Dementia; EO: Early onset; EOAD: Early onset Alzheimer's disease;

FDG: Fluorodeoxyglucose; FWE: Family-wise error; FWHM: Full-width halfmax; GMD: Gray matter density; HS: Hippocampal sclerosis; ICV: Intracranial volume; LO: Late onset; LOAD: Late onset Alzheimer's disease; LATE: Limbicpredominant age-related TDP-43 encephalopathy; MCI: Mild cognitive impairment; MMSE: Mini-mental state exam; MNI: Montreal Neurological Institute; PART: Primary age-related tauopathy; nonAD: Amyloid negative; SUVR: Standardized uptake value ratio; TDP-43: TAR-DNA binding protein 43

\section{Acknowledgements}

Data used in preparation of this article were obtained from the Alzheimer's Disease Neuroimaging Initiative (ADNI) database (adni.loni.usc.edu). As such, the investigators within the ADNI contributed to the design and implementation of ADNI and/or provided data but did not participate in analysis or writing of this report. A complete listing of ADNI investigators can be found at: http://adni.loni.usc.edu/wpcontent/uploads/how_to_apply/ ADNI_Acknowledgement_List.pdf

\section{Authors' contributions}

ECS was the primary author responsible for drafting the manuscript and participated in all stages of data analysis and manuscript preparation. DS assisted with some of the analyses and took part in manuscript preparation and revision. MP assisted with some of the analyses and took part in manuscript preparation and revision. VHC with some of the analyses and took part in manuscript preparation and revision. TD assisted with some of the analyses and took part in manuscript preparation and revision. NG assisted with some of the analyses and took part in manuscript preparation and revision. SLR assisted with image processing and took part in manuscript preparation and revision. AJS assisted with data interpretation and took part in manuscript preparation and revision. LGA was responsible for the study concept and design. She provided scientific oversight over all analyses, result interpretation, and participated in all stages of manuscript preparation. The authors read and approved the final manuscript.

\section{Authors' information}

Not applicable.

\section{Funding}

The analyses reported in this manuscript were funded by the NIA R56 AG057195, NIA U01 AG057195, NIA R01 AG040770, NIA K02 AG048240, NIA P30 AG010133, and NIA K01 AG049050.

Data collection and sharing for this project was funded by the Alzheimer's Disease Neuroimaging Initiative (ADNI) (National Institutes of Health Grant U01 AG024904) and DOD ADNI (Department of Defense award number W81XWH-12-2-0012). ADNI is funded by the National Institute on Aging, the National Institute of Biomedical Imaging and Bioengineering, and through generous contributions from the following: Alzheimer's Association; Alzheimer's Drug Discovery Foundation; BioClinica, Inc.; Biogen Idec Inc.; Bristol- 
Myers Squibb Company; Eisai Inc.; Elan Pharmaceuticals, Inc.; Eli Lilly and Company; F. Hoffmann-La Roche Ltd. and its affiliated company Genentech, Inc;; GE Healthcare; Innogenetics, N.V.; IXICO Ltd.; Janssen Alzheimer Immunotherapy Research \& Development, LLC.; Johnson \& Johnson Pharmaceutical Research \& Development LLC.; Medpace, Inc.; Merck \& Co., Inc;; Meso Scale Diagnostics, LLC.; NeuroRx Research; Novartis Pharmaceuticals Corporation; Pfizer Inc;; Piramal Imaging; Servier; Synarc Inc.; and Takeda Pharmaceutical Company. The Canadian Institutes of Health Research is providing funds to support ADNI clinical sites in Canada. Private sector contributions are facilitated by the Foundation for the National Institutes of Health (www.fnih.org). The grantee organization is the Northern California Institute for Research and Education, and the study is coordinated by the Alzheimer's Disease Cooperative Study at the University of California, San Diego. ADNI data are disseminated by the Laboratory for Neuro Imaging at the University of Southern California.

\section{Availability of data and materials}

All data presented is available through the ADNI website at http://adni.loni. usc.edu/data-samples/access-data/. Investigators may apply for access by filling out the online application form and adhering to the data use agreement.

\section{Ethics approval and consent to participate}

All procedures were approved by the Institutional Review Boards of all participating institutions. Written informed consent was obtained from every research participant according to the Declaration of Helsinki and the Belmont Report. The analyses reported here were conducted under Indiana University IRB protocol \#1506941363 (exempt).

\section{Consent for publication}

Not applicable.

\section{Competing interests}

Eddie Stage, PhD, reports no competing interests. Meredith Phillips, MS, reports no competing interests. Victor Hugo Canela, MS, reports no competing interests. Tugce Duran, BS, reports no competing interests. Naira Goukasian, BS, reports no competing interests. Shannon L. Risacher, PhD, reports no competing interests. Andrew J. Saykin, PsyD, has received research support from Eli Lilly and AVID Radiopharmaceuticals.

Liana G. Apostolova, MD, MS, has served on an Advisory Board for Eli Lilly and Biogen and on the Speakers Bureau for Piramal and Eli Lilly.

\section{Author details}

'Department of Neurology, Indiana University School of Medicine, 355 W 16th Street, Suite 4022, Indianapolis, IN 46202, USA. ${ }^{2}$ Clinical Imaging, Eli Lilly and Company, Indianapolis, IN, USA. ${ }^{3}$ Department of Epidemiology and Biostatistics, Indiana University School of Public Health, Bloomington, IN, USA. ${ }^{4}$ Department of Biomedical Sciences Graduate School, Wake Forest University School of Medicine, Winston Salem, NC, USA. ${ }^{5}$ University of Vermont College of Medicine, Burlington, VT, USA. ${ }^{6}$ Department of Radiology and Imaging Sciences, Center for Neuroimaging, Indiana University School of Medicine, Indianapolis, IN, USA. 'Department of Medical and Molecular Genetics, Indiana University School of Medicine, Indianapolis, IN, USA. ${ }^{8}$ Indiana University Network Science Institute, Indianapolis, IN, USA.

Received: 24 October 2019 Accepted: 23 June 2020 Published online: 05 August 2020

\section{References}

1. Alzheimer Association. 2019 Alzheimer's disease facts and figures. Alzheimers Dement. 2019;15(3):321-87.

2. Beach TG, Monsell SE, Phillips LE, Kukull W. Accuracy of the clinical diagnosis of Alzheimer disease at National Institute on Aging Alzheimer Disease Centers, 2005-2010. J Neuropathol Exp Neurol. 2012; 71(4):266-73.

3. van der Flier WM, Pijnenburg YA, Fox NC, Scheltens P. Early-onset versus late-onset Alzheimer's disease: the case of the missing APOE varepsilon4 allele. Lancet Neurol. 2011;10(3):280-8.
4. Konijnenberg E, Fereshtehnejad SM, Kate MT, Eriksdotter M, Scheltens P Johannsen $\mathrm{P}$, et al. Early-onset dementia: frequency, diagnostic procedures, and quality indicators in three European tertiary referral centers. Alzheimer Dis Assoc Disord. 2017:31(2):146-51.

5. Katzman R. Editorial: The prevalence and malignancy of Alzheimer disease. A major killer. Arch Neurol. 1976;33(4):217-8.

6. Bird TD, Stranahan S, Sumi SM, Raskind M. Alzheimer's disease: choline acetyltransferase activity in brain tissue from clinical and pathological subgroups. Ann Neurol. 1983;14(3):284-93.

7. Rossor MN, Iversen LL, Reynolds GP, Mountjoy CQ, Roth M. Neurochemical characteristics of early and late onset types of Alzheimer's disease. Br Med J (Clin Res Ed). 1984;288(6422):961-4.

8. Marshall GA, Fairbanks LA, Tekin S, Vinters HV, Cummings JL. Early-onset Alzheimer's disease is associated with greater pathologic burden. J Geriatr Psychiatry Neurol. 2007;20(1):29-33.

9. Murray ME, Graff-Radford NR, Ross OA, Petersen RC, Duara R, Dickson DW. Neuropathologically defined subtypes of Alzheimer's disease with distinct clinical characteristics: a retrospective study. Lancet Neurol. 2011;10(9):785-96.

10. Sakamoto S, Ishii K, Sasaki M, Hosaka K, Mori T, Matsui M, et al. Differences in cerebral metabolic impairment between early and late onset types of Alzheimer's disease. J Neurol Sci. 2002;200(1-2):27-32.

11. Migliaccio R, Agosta F, Possin KL, Canu E, Filippi M, Rabinovici GD, et al. Mapping the progression of atrophy in early- and late-onset Alzheimer's disease. J Alzheimers Dis. 2015;46(2):351-64.

12. Lehmann M, Ghosh PM, Madison C, Laforce R Jr, Corbetta-Rastelli C, Weiner $M W$, et al. Diverging patterns of amyloid deposition and hypometabolism in clinical variants of probable Alzheimer's disease. Brain. 2013;136(Pt 3):844-58.

13. Scholl M, Ossenkoppele R, Strandberg O, Palmqvist S, Swedish Bio Fs, Jogi J, et al. Distinct 18F-AV-1451 tau PET retention patterns in early- and lateonset Alzheimer's disease. Brain 2017;140(9):2286-2294.

14. Whitwell JL, Martin P, Graff-Radford J, Machulda MM, Senjem ML, Schwarz CG, et al. The role of age on tau PET uptake and gray matter atrophy in atypical Alzheimer's disease. Alzheimers Dement. 2019;15:675-85.

15. Smits LL, Pijnenburg YA, van der Vlies AE, Koedam EL, Bouwman FH, Reuling IE, et al. Early onset APOE E4-negative Alzheimer's disease patients show faster cognitive decline on non-memory domains. Eur Neuropsychopharmacol. 2015;25(7):1010-7.

16. Koedam EL, Lauffer $V$, van der Vlies AE, van der Flier WM, Scheltens $P$, Pijnenburg YA. Early-versus late-onset Alzheimer's disease: more than age alone. J Alzheimers Dis. 2010:19(4):1401-8.

17. Smits LL, Pijnenburg YA, Koedam EL, van der Vlies AE, Reuling IE, Koene T, et al. Early onset Alzheimer's disease is associated with a distinct neuropsychological profile. J Alzheimers Dis. 2012;30(1):101-8.

18. Moller C, Vrenken H, Jiskoot L, Versteeg A, Barkhof F, Scheltens $P$, et al. Different patterns of gray matter atrophy in early-and late-onset Alzheimer's disease. Neurobiol Aging. 2013;34(8):2014-22.

19. Petersen RC, Aisen PS, Beckett LA, Donohue MC, Gamst AC, Harvey DJ, et al. Alzheimer's Disease Neuroimaging Initiative (ADNI): clinical characterization. Neurology. 2010;74(3):201-9.

20. McKhann G, Drachman D, Folstein M, Katzman R, Price D, Stadlan EM. Clinical diagnosis of Alzheimer's disease: report of the NINCDS-ADRDA Work Group under the auspices of Department of Health and Human Services Task Force on Alzheimer's Disease. Neurology. 1984;34(7):939-44.

21. Folstein MF, Folstein SE, McHugh PR. "Mini-mental state". A practical method for grading the cognitive state of patients for the clinician. J Psychiatr Res. 1975;12(3):189-98.

22. Morris JC. The Clinical Dementia Rating (CDR): current version and scoring rules. Neurology. 1993;43(11):2412-4.

23. D. W. Wechsler Memory Scale - Revised, vol. 1987. San Antonio: Psychological Corporation; 1987.

24. Fleisher AS, Chen K, Liu X, Roontiva A, Thiyyagura P, Ayutyanont N, et al. Using positron emission tomography and florbetapir F18 to image cortical amyloid in patients with mild cognitive impairment or dementia due to Alzheimer disease. Arch Neurol. 2011;68(11):1404-11.

25. Shaw LM, Vanderstichele H, Knapik-Czajka M, Clark CM, Aisen PS, Petersen RC, et al. Cerebrospinal fluid biomarker signature in Alzheimer's disease neuroimaging initiative subjects. Ann Neurol. 2009;65(4):403-13.

26. Risacher SL, Kim S, Shen L, Nho K, Foroud T, Green RC, et al. The role of apolipoprotein $\mathrm{E}$ (APOE) genotype in early mild cognitive impairment (E$\mathrm{MCl})$. Front Aging Neurosci. 2013;5:11. 
27. Jagust WJ, Bandy D, Chen K, Foster NL, Landau SM, Mathis CA, et al. The Alzheimer's Disease Neuroimaging Initiative positron emission tomography core. Alzheimers Dement. 2010;6(3):221-9.

28. Clark CM, Pontecorvo MJ, Beach TG, Bedell BJ, Coleman RE, Doraiswamy PM, et al. Cerebral PET with florbetapir compared with neuropathology at autopsy for detection of neuritic amyloid-beta plaques: a prospective cohort study. Lancet Neurol. 2012;11(8):669-78.

29. Shcherbinin S, Schwarz AJ, Joshi A, Navitsky M, Flitter M, Shankle WR, et al Kinetics of the tau PET tracer 18F-AV-1451 (T807) in subjects with normal cognitive function, mild cognitive impairment, and Alzheimer disease. J Nucl Med. 2016;57(10):1535-42

30. Jellinger KA, Alafuzoff I, Attems J, Beach TG, Cairns NJ, Crary JF, et al. PART, a distinct tauopathy, different from classical sporadic Alzheimer disease. Acta Neuropathol. 2015;129(5):757-62.

31. James BD, Wilson RS, Boyle PA, Trojanowski JQ, Bennett DA, Schneider JA TDP-43 stage, mixed pathologies, and clinical Alzheimer's-type dementia. Brain. 2016;139(11):2983-93.

32. Nelson PT, Dickson DW, Trojanowski JQ, Jack CR, Boyle PA, Arfanakis K, et al. Limbic-predominant age-related TDP-43 encephalopathy (LATE): consensus working group report. Brain. 2019;142(6):1503-27.

33. Lowe VJ, Curran G, Fang P, Liesinger AM, Josephs KA, Parisi JE, et al. An autoradiographic evaluation of AV-1451 tau PET in dementia. Acta Neuropathol Commun. 2016;4(1):58

34. Wren MC, Lashley T, Arstad E, Sander K. Large inter- and intra-case variability of first generation tau PET ligand binding in neurodegenerative dementias. Acta Neuropathol Commun. 2018;6(1):34.

35. McAleese KE, Walker L, Erskine D, Thomas AJ, McKeith IG, Attems J. TDP-43 pathology in Alzheimer's disease, dementia with Lewy bodies and ageing. Brain Pathol. 2017;27(4):472-9.

36. Josephs KA, Dickson DW, Tosakulwong N, Weigand SD, Murray ME, Petrucelli $L$, et al. Rates of hippocampal atrophy and presence of postmortem TDP-43 in patients with Alzheimer's disease: a longitudinal retrospective study. Lancet Neurol. 2017;16(11):917-24.

37. Mackenzie IR, Rademakers R, Neumann M. TDP-43 and FUS in amyotrophic lateral sclerosis and frontotemporal dementia. Lancet Neurol. 2010;9(10): 995-1007.

38. Arai T, Hasegawa M, Akiyama H, Ikeda K, Nonaka T, Mori H, et al. TDP-43 is a component of ubiquitin-positive tau-negative inclusions in frontotemporal lobar degeneration and amyotrophic lateral sclerosis. Biochem Biophys Res Commun. 2006:351(3):602-11.

39. Nag S, Yu L, Capuano AW, Wilson RS, Leurgans SE, Bennett DA, et al. Hippocampal sclerosis and TDP-43 pathology in aging and Alzheimer disease. Ann Neurol. 2015;77(6):942-52.

40. Josephs KA, Whitwell JL, Knopman DS, Hu WT, Stroh DA, Baker M, et al. Abnormal TDP-43 immunoreactivity in AD modifies clinicopathologic and radiologic phenotype. Neurology. 2008;70(19 Pt 2):1850-7.

41. Wilson RS, Yu L, Trojanowski JQ, Chen EY, Boyle PA, Bennett DA, et al. TDP43 pathology, cognitive decline, and dementia in old age. JAMA Neurol. 2013;70(11):1418-24.

42. Nag S, Yu L, Wilson RS, Chen EY, Bennett DA, Schneider JA. TDP-43 pathology and memory impairment in elders without pathologic diagnoses of AD or FTLD. Neurology. 2017:88(7):653-60.

43. Botha $H$, Mantyh WG, Murray ME, Knopman DS, Przybelski SA, Wiste $\mathrm{HJ}$, et al. FDG-PET in tau-negative amnestic dementia resembles that of autopsy-proven hippocampal sclerosis. Brain. 2018;141(4):1201-17.

44. Grothe M. Towards a topographic imaging biomarker of TDP-43 pathology in amnestic dementia: patient stratification based on FDG-PET patterns in autopsy-confirmed cases. [Human Amyloid Imaging 2019 Presentation]. In press 2019.

45. Bejanin A, Murray ME, Martin P, Botha H, Tosakulwong N, Schwarz CG, et al. Antemortem volume loss mirrors TDP-43 staging in older adults with non-frontotemporal lobar degeneration. Brain. 2019;142:3621-35.

46. Rascovsky K, Hodges JR, Knopman D, Mendez MF, Kramer JH, Neuhaus $J$, et al. Sensitivity of revised diagnostic criteria for the behavioural variant of frontotemporal dementia. Brain. 2011;134(Pt 9):2456-77.

47. Baborie A, Griffiths TD, Jaros E, McKeith IG, Burn DJ, Richardson A, et al. Pathological correlates of frontotemporal lobar degeneration in the elderly. Acta Neuropathol. 2011:121(3):365-71.

\section{Publisher's Note}

Springer Nature remains neutral with regard to jurisdictional claims in published maps and institutional affiliations.

\section{Ready to submit your research? Choose BMC and benefit from:}

- fast, convenient online submission

- thorough peer review by experienced researchers in your field

- rapid publication on acceptance

- support for research data, including large and complex data types

- gold Open Access which fosters wider collaboration and increased citations

- maximum visibility for your research: over $100 \mathrm{M}$ website views per year

At BMC, research is always in progress.

Learn more biomedcentral.com/submissions 